\title{
AN APPROXIMATION THEORY FOR OSCILLATIONS OF DIFFERENTIAL EQUATIONS ${ }^{1}$
}

\author{
JOHN GREGORY
}

ABSTRACT. In an earlier paper we gave an approximation theory of focal points and focal intervals. The fundamental purpose of this paper is to show that the ideas and methods of that paper can be used to give an approximation theory for oscillation for linear selfadjoint differential (and integral-differential) equations. These methods follow from the theory of quadratic forms given by Hestenes.

In $\S 1$ we give the preliminaries needed for this paper. In $\S 2$ we define oscillation for quadratic control problems and discuss their connection with differential equations. In $\S 3$ we give our main approximation results relating the oscillation points $\lambda_{n}\left(\sigma_{0}\right)$ $(n=i, 2,3, \cdots)$ for a given problem to the oscillation points $\lambda_{n}(\sigma)$ $(n=1,2,3, \cdots)$ of an approximating problem. The element $\sigma$ belongs to a metric space $(\Sigma, d)$. The main result is to show that the $n$th oscillation point $\lambda_{n}(\sigma)$ is a continuous function of $\sigma(n=i$, $2,3, \cdots)$. For completeness in $\S 4$ we present an example for fourth order differential equations where the approximation is by discrete problems. Thus oscillation points can be "easily" computed by numerical algorithms.

1. Preliminaries. In this section we give the approximation hypothesis, the focal point hypothesis, and basic results required for the remainder of this paper. $\mathscr{A}$ will denote a real Hilbert space with inner product $(x, y)$ and norm $\|x\|=(x, x)^{1 / 2}$. Strong convergence is denoted by $x_{q} \Rightarrow x_{0}$ and weak convergence by $x_{q} \rightarrow x_{0}$. For the sake of convenience the next three paragraphs describe properties that occur throughout this paper.

Let $\Sigma$ be a metric space with metric $\rho$. A sequence $\left\{\sigma_{r}\right\}$ in $\Sigma$ converges to $\sigma_{0}$ in $\Sigma$, written $\sigma_{r} \rightarrow \sigma_{0}$, if $\lim _{r=\infty} \rho\left(\sigma_{r}, \sigma_{0}\right)=0$. For each $\sigma$ in $\Sigma$ let $\mathscr{A}(\sigma)$ be a closed subspace of $\mathscr{A}$ such that

(1a) if $\sigma_{r} \rightarrow \sigma_{0}, x_{r}$ in $\mathscr{A}\left(\sigma_{r}\right), x_{r} \rightarrow y_{0}$ then $y_{0}$ is in $\mathscr{A}\left(\sigma_{0}\right)$;

(1b) if $x_{0}$ is in $\mathscr{A}\left(\sigma_{0}\right)$ and $\varepsilon>0$ there exists $\delta>0$ such that whenever $\rho\left(\sigma, \sigma_{0}\right)<\delta$, there exists $x_{\sigma}$ in $\mathscr{A}(\sigma)$ satisfying $\left\|x_{0}-x_{\sigma}\right\|<\varepsilon$.

Received by the editors May 22, 1972 and, in revised form, December 22, 1972.

AMS (MOS) subject classifications (1970). Primary 34C10, 49G99, 41A65.

Key words and phrases. Approximation theory, oscillation, quadratic forms, Hilbert space, conjugate points, calculus of variations.

${ }^{1}$ The preparation of this paper was sponsored in part by the National Research Council of Canada, Grant No. A8045.

(c) American Mathematical Society 1973 
For each $\sigma$ in $\Sigma$ let $J(x ; \sigma)$ be a quadratic form defined on $\mathscr{A}(\sigma)$ with $J(x, y ;)$ the associated bilinear form. For $r=0,1,2, \cdots$ let $x_{r}$ be in $\mathscr{A}\left(\sigma_{r}\right)$, $y_{r}$ in $\mathscr{A}\left(\sigma_{r}\right)$ such that: if $x_{r} \rightarrow x_{0}, y_{r} \Rightarrow y_{0}$ and $\sigma_{r} \rightarrow \sigma_{0}$ then

$$
\begin{gathered}
\lim _{r=\infty} J\left(x_{r}, y_{r} ; \sigma_{r}\right)=J\left(x_{0}, y_{0} ; \sigma_{0}\right) ; \\
\liminf _{r=\infty} J\left(x_{r} ; \sigma_{r}\right) \geqq J\left(x_{0} ; \sigma_{0}\right) ; \quad \text { and } \\
\lim _{r=\infty} J\left(x_{r} ; \sigma_{r}\right)=J\left(x_{0} ; \sigma_{0}\right) \quad \text { implies } x_{r} \Rightarrow x_{0} .
\end{gathered}
$$

Let $a, b$ be real numbers $(a<b)$ and define $\Lambda=[a, b]$. Let $\{\mathscr{H}(\lambda): \lambda$ in $\Lambda\}$ be a one-parameter family of closed subspaces of $\mathscr{A}$ such that $\mathscr{H}(a)=0$, $\mathscr{H}(b)=\mathscr{A}$, and $\mathscr{H}\left(\lambda_{1}\right) \subset \mathscr{H}\left(\lambda_{2}\right)$ whenever $\lambda_{1}, \lambda_{2}$ in $\Lambda, \lambda_{1}<\lambda_{2}$. In this paper we will require one or both of the additional hypotheses:

$$
\begin{aligned}
& \mathscr{H}\left(\lambda_{0}\right)=\bigcap_{\lambda_{0}<\lambda \leqq b} \mathscr{H}(\lambda) \quad \text { whenever } a \leqq \lambda_{0}<b, \text { and } \\
& \mathscr{H}\left(\lambda_{0}\right)=\bigcup_{\alpha \leqq \lambda<\lambda_{0}} \mathscr{H}(\lambda)
\end{aligned}
$$

is satisfied. We note that $\bar{S}$ denotes the closure of $S$.

The signature (index) of a quadratic form $Q(x)$ on a subspace $\mathscr{B}$ of $\mathscr{A}$ is the dimension of a maximal, linear subclass $\mathscr{C}$ of $\mathscr{B}$ such that $x \neq 0$ in $\mathscr{C}$ implies $Q(x)<0$. The nullity of $Q(x)$ on $\mathscr{B}$ is the dimension of the set $\mathscr{B}_{0}=\{x$ in $\mathscr{B} \mid Q(x, y)=0$ for all $y$ in $\mathscr{B}\}$. The vector $x$ is said to be a $Q$ null vector of $\mathscr{B}$.

We now define the spaces $\mathscr{B}(\mu)$ which "resolve" the space $\mathscr{A}(\sigma)$. Inequality results are then given relating the signatures $s(\mu)$ and $n(\mu)$ to $s\left(\mu_{0}\right)$ and $n\left(\mu_{0}\right)$. Theorems 1,2 , and 3 are proven in [2].

Let $M=\Lambda \times \Sigma$ be the metric space with metric $d$ defined by $d\left(\mu_{1}, \mu_{2}\right)=$ $\left|\lambda_{2}-\lambda_{1}\right|+\rho\left(\sigma_{2}, \sigma_{1}\right)$ where $\mu_{1}=\left(\lambda_{1}, \sigma_{1}\right)$ and $\mu_{2}=\left(\lambda_{2}, \sigma_{2}\right)$. For each $\mu=(\lambda, \sigma)$ in $M$ define $J(x ; \mu)=J(x ; \sigma)$ on the space $\mathscr{B}(\mu)=\mathscr{A}(\sigma) \cap \mathscr{H}(\lambda)$. Let $s(\mu)=s(\lambda, \sigma), n(\mu)=n(\lambda, \sigma)$ denote the index and nullity of $J(x ; \mu)$ on $\mathscr{B}(\mu)$.

TheOREM 1. Assume (1a) and (2) hold and that (3a) holds. For any $\mu_{0}=\left(\lambda_{0}, \sigma_{0}\right)$ in $M$ there exists $\delta>0$ such that if $\mu=(\lambda, \sigma), d\left(\mu_{0}, \mu\right)<\delta$ then

$$
s(\lambda, \sigma)+n(\lambda, \sigma) \leqq s\left(\lambda_{0}, \sigma_{0}\right)+n\left(\lambda_{0}, \sigma_{0}\right) .
$$

Theorem 2. Assume (1b) and (2) hold and that (3b) holds. For any $\mu_{0}=\left(\lambda_{0}, \sigma_{0}\right)$ in $M$ there exists $\delta>0$ such that if $\mu=(\lambda, \sigma), d\left(\mu_{0}, \mu\right)<\delta$ then

$$
s\left(\lambda_{0}, \sigma_{0}\right) \leqq s(\lambda, \sigma) .
$$


Combining Theorems 1 and 2 we have:

THEOREM 3. For any $\mu_{0}=\left(\lambda_{0}, \sigma_{0}\right)$ in $M$ there exists $\delta>0$ such that if $\mu=(\lambda, \sigma), d\left(\mu_{0}, \mu\right)<\delta$ then

(6) $s\left(\lambda_{0}, \sigma_{0}\right) \leqq s(\lambda, \sigma) \leqq s(\lambda, \sigma)+n(\lambda, \sigma) \leqq s\left(\lambda_{0}, \sigma_{0}\right)+n\left(\lambda_{0}, \sigma_{0}\right)$.

\section{Furthermore}

$$
n\left(\lambda_{0}, \sigma_{0}\right)=0 \text { implies } s(\lambda, \sigma)=s\left(\lambda_{0}, \sigma_{0}\right) \text { and } n(\lambda, \sigma)=0 .
$$

2. Oscillation for quadratic control problems. In this section we give a new definition of oscillation. It is immediate that this definition reduces to the usual definition for the usual (normal second order) problems. It appears that this definition is the natural one to use for higher order quadratic control and integral equation problems, approximation results, eigenvalue problems, quantitative results, and more abstract systems. Thus let

where

$$
J(x)=\int_{a}^{b} 2 \omega(t, x, u) d t
$$

$$
2 \omega(t, x, u)=x^{*} P(t) x+x^{*} Q(t) u+u^{*} Q^{*}(t) x+u^{*} R(t) u
$$

is given relative to a one-parameter family $\mathscr{H}(\lambda)(a \leqq \lambda \leqq b)$ of $\operatorname{arcs}(x, u)$ satisfying the control equation

$$
\dot{x}=A x+B u \quad(a \leqq t \leqq \lambda),
$$

a linear constraint

$$
M x+N u=0 \quad(a \leqq t \leqq \lambda)
$$

and the boundary conditions

$$
\begin{gathered}
x(a)=0, \\
x(t)=0, \quad u(t)=0 \quad(\lambda \leqq t \leqq b) .
\end{gathered}
$$

In the above $x(t)$ is absolutely continuous; $\dot{x}(t)$ and $u(t)$ are square integrable; $A, P=P^{*}$, and $Q$ are square integrable; $B, M, N$ and $R=R^{*}$ are essentially bounded and measurable. The vectors $x$ and $u$ are respectively $n$ and $q$ dimensional while the symbol "*" denotes matrix transpose. In addition we assume $J(x)$ is elliptic in the sense of Hestenes (see [1]). The following theorem is given in [4].

THEOREM 4. The vector $x$ is $J$ orthogonal to $\mathscr{H}(\lambda)$ if and only if there exists an absolutely continuous vector $p(t)(a \leqq t \leqq \lambda)$ and a square integrable 
vector $v(t)(a \leqq t \leqq \lambda)$ such that

$$
\dot{p}+A^{*} p+M^{*} v=\omega_{x}
$$

and

$$
B^{*} p+N^{*} v=\omega_{u}
$$

hold on $a \leqq t \leqq \lambda$. Thus $x$ is in $\mathscr{H}_{0}(\lambda)$ if and only if (9) and (10) hold and (11) holds on $a \leqq t \leqq \lambda$.

The control problem gives by (8), (9) and (10) is oscillatory of degree $m$ if $s(b)=m$. Equation (11) is oscillatory of degree $m$ on $a \leqq t \leqq b$ if there exist vectors $(x, u, p, v)$ satisfying the above continuity conditions, such that $(x, u)$ is $J$ orthogonal to $\mathscr{H}(b)$ and the well defined associated control problem is oscillatory of degree $m$. In this case the vector $(x, u)$ is an oscillatory solution of degree $m$ on $a \leqq t \leqq b$. Equation (11) is oscillatory if for any integer $M>0$ there exist real numbers $a<b$ such that equation (11) is oscillatory of degree $m$ on $a \leqq t \leqq b$ and $m \geqq M$. Finally equation (11) is nonoscillatory if it is not oscillatory.

Since the reader may be lost, we remark that the "simplest" quadratic form represented by (8) and (9) is $J(x)=\int_{a}^{b}\left[r(t) x^{\prime 2}-p(t) x^{2}\right] d t$ whose associated Euler's equation is $\left(r x^{\prime}\right)^{\prime}+p x=0$. We assume $r(t)>0, r(t)$ and $p(t)$ are piecewise continuous, and that primes denote derivates. The signature $s(\lambda)$ increases by one at each point $\lambda(a<\lambda<b)$ for which a nonzero solution of the second order differential equation vanishes at both $t=a$ and $t=\lambda$. The nullity $n(\lambda)=1$ at such points and equals zero at all other points.

We note that these procedures can be used for quadratic forms such as

$$
J(x)=\int_{a}^{b} R_{\alpha \beta}^{i j}(t) x_{\alpha}^{(i)}(t) x_{\beta}^{j}(t) d t \quad(\alpha, \beta=1, \cdots, p ; i, j=0, \cdots, n)
$$

whose associated Euler's equations are integral-differential equations and for quadratic forms associated with partial differential equations.

3. Oscillation points and oscillation intervals. Assume for each $\sigma$ in $\Sigma$ we define

where

$$
J(x ; \sigma)=\int_{a}^{b} 2 \omega_{\sigma}(t, x, u) d t
$$

$$
2 \omega_{\sigma}=x^{*} P_{\sigma}(t) x+x^{*} Q_{\sigma}(t) u+u^{*} Q_{\sigma}^{*}(t) x+u^{*} R_{\sigma}(t) u
$$

is given with arcs $(x, u)$ in $(\sigma)$ satisfying the control equations

$$
\begin{array}{ll}
\dot{x}=A_{\sigma} x+B_{\sigma} u & (a \leqq t \leqq b), \\
M_{\sigma} x+N_{\sigma} u=0 & (a \leqq t \leqq b),
\end{array}
$$


and the boundary conditions (10) with $\lambda=b$. Furthermore, assume that $R_{\sigma}(t), M_{\sigma}(t), A_{\sigma}(t)$ etc. satisfy the conditions in $\$ 2$ for $R(t), M(t), A(t)$ etc. and that (1) and (2) hold. Let $B(\lambda, \sigma)$ be the set of all arcs $(x, u)$ satisfying (9) ${ }_{\sigma}$ and (10) with $A_{\sigma}, B_{\sigma}, M_{\sigma}$, and $N_{\sigma}$ respectively replacing $A, B, M, N$ in (9) and (10). Let $s(\lambda, \sigma)$ and $n(\lambda, \sigma)$ be the respective index and nullity of $J(x ; \sigma)$ on $B(\lambda, \sigma)$.

Let $\sigma_{0}$ in $\Sigma$ be given. A point $\lambda_{0}$ at which $s\left(\lambda, \sigma_{0}\right)$ is discontinuous will be called an oscillation point of $J\left(x ; \sigma_{0}\right)$ relative to $\{H(\lambda): \lambda$ in $\Lambda\}$. The difference $s\left(\lambda_{0}+0, \sigma_{0}\right)-s\left(\lambda_{0}, \sigma_{0}\right)$ will be called the order of $\lambda_{0}$ as an oscillation point (of $\sigma_{0}$ ). An oscillation point $\lambda_{0}$ is counted the number of times equal to its order. In the above $s\left(\lambda_{0}+0, \sigma_{0}\right)$ is the right-hand limit of $s(\lambda, \sigma)$ as $\lambda \rightarrow \lambda_{0}$ from above. The quantity $s\left(\lambda_{0}-0, \sigma_{0}\right)$ is similarly defined. Thus $x$ is in $B(\lambda, \sigma)$ if and only if the following equations hold in the " $\sigma$ " notation $(9)_{\sigma}$, (10) and (11) on $a \leqq t \leqq \lambda$.

It has been shown in [1] that (3b) implies $s\left(\lambda-0, \sigma_{0}\right)=s\left(\lambda, \sigma_{0}\right)$ while (3a) and the disjoint hypothesis of Theorem 5 imply $s\left(\lambda+0, \sigma_{0}\right)=s\left(\lambda, \sigma_{0}\right)$. Thus if $\lambda_{n}(\sigma)(n=1,2,3, \cdots)$ is the $n$th oscillation point with respect to $\sigma$ we have

THEOREM 5. Assume (3) holds. Let $\sigma_{0}$ in $\Sigma$ be given such that $\lambda^{\prime}, \lambda^{\prime \prime}$ in $\Lambda$, $a \leqq \lambda^{\prime}<\lambda^{\prime \prime} \leqq b$ imply the $J\left(x ; \sigma_{0}\right)$ null vectors on $\mathscr{B}\left(\lambda^{\prime}, \sigma_{0}\right)$ and $\mathscr{B}\left(\lambda^{\prime \prime}, \sigma_{0}\right)$ are disjoint. Assume $\lambda^{\prime}$ and $\lambda^{\prime \prime}$ are not oscillation points of $\sigma_{0}\left(a \leqq \lambda^{\prime}<\lambda^{\prime \prime}<b\right)$ and there exist exactly $k$ oscillation points of $\sigma_{0}$ on $\left(\lambda^{\prime}, \lambda^{\prime \prime}\right)$. Then there exists $\varepsilon>0$ such that $\rho\left(\sigma, \sigma_{0}\right)<\varepsilon$ implies there are exactly $k$ oscillation points of $\sigma$ on $\left(\lambda^{\prime}, \lambda^{\prime \prime}\right)$.

In fact, if $\lambda_{n}\left(\sigma_{0}\right) \leqq \lambda_{n+1}\left(\sigma_{0}\right) \leqq \cdots \leqq \lambda_{n+k-1}\left(\sigma_{0}\right)$ are the $k$ oscillation points of $\sigma_{0}$ on $\left(\lambda^{\prime}, \lambda^{\prime \prime}\right)$ then $\lambda_{n}(\sigma) \leqq \lambda_{n+1}(\sigma) \leqq \cdots \leqq \lambda_{n+k-1}(\sigma)$ are the $k$ oscillation points of $\sigma$ on $\left(\lambda^{\prime}, \lambda^{\prime \prime}\right)$.

Assume $s\left(\lambda^{\prime}, \sigma_{0}\right)=n$, then by the above remark $s\left(\lambda^{\prime \prime}, \sigma_{0}\right)=n+k-1$ and $n\left(\lambda^{\prime}, \sigma_{0}\right)=n\left(\lambda^{\prime \prime}, \sigma_{0}\right)=0$. By (7) there exists $\delta>0$ such that if $\rho\left(\sigma, \sigma_{0}\right)<\delta$ then $n\left(\lambda^{\prime}, \sigma\right)=n\left(\lambda^{\prime \prime}, \sigma\right)=0, s\left(\lambda^{\prime}, \sigma\right)=n, s\left(\lambda^{\prime \prime}, \sigma\right)=n+k-1$. The result follows by definition.

COROLlaRy 6. Under the above hypothesis there exists $\varepsilon>0$ such that $\rho\left(\sigma, \sigma_{0}\right)<\varepsilon$ and $a \leqq \lambda \leqq a+\varepsilon$ imply there exists no oscillation point $\lambda$ of $\sigma$.

COROLLARY 7. Under the above hypothesis the nth oscillation point $\lambda_{n}(\sigma)$ is a continuous function of $\sigma(n=1,2,3, \cdots)$.

If we assume that the disjoint hypothesis of Theorem 5 does not hold we obtain an oscillation interval theory. In this case condition (3) implies that if $x_{0}$ is a $J\left(x ; \sigma_{0}\right)$ null vector of $\mathscr{B}\left(\lambda_{0}, \sigma_{0}\right)$ then $\sigma_{0}$ belongs to a proper closed subinterval $\Lambda_{1}=\left[\lambda^{\prime}, \lambda^{\prime \prime}\right]$ of $\Lambda$ where $\Lambda_{1}=\left\{\lambda\right.$ in $\Lambda: x_{0}$ is a $J\left(x ; \sigma_{0}\right)$ null vector of $\left.\mathscr{B}\left(\lambda, \sigma_{0}\right)\right\}$. [2] shows that oscillation intervals can be well defined in the 
same way as focal intervals. They can also be counted exactly as the focal intervals in [2]. Oscillation intervals are important as oscillation points are the endpoints $\lambda^{\prime \prime}$ of the interval $\Lambda$, described above.

4. An example. For ease of presentation we will consider the quadratic form $J(x)=\int_{a}^{b}\left[r(t) x^{\prime \prime 2}-p(t) x^{2}\right] d t$ which is associated with the fourth order equation $\left(r x^{\prime \prime}\right)^{\prime \prime}-p x=0$. The fundamental Hilbert space is the set of arcs $x(t)$ satisfying $x \in C^{\prime}[a, b], x^{\prime}$ is absolutely continuous and $x^{\prime \prime} \in L^{2}[a, b]$. In this example we consider the discrete approximation case. Thus define $J(x ; 0)=J(x)$ and $\mathscr{A}(0)$ to be the Hilbert space of functions satisfying the above conditions such that $x(a)=x^{\prime}(a)=x^{\prime}(b)=x^{\prime}(b)=0$.

The general $2 n$th order case (in our example $n=2$ ) is an immediate modification of this example and is left to the reader. Note that our procedures give an efficient algorithm for numerical calculation of oscillation points.

For $\sigma=1 / n$ define the partition

$$
\pi(\sigma)=\left\{a_{k}=a+k(b-a) / n \mid k=0,1, \cdots, n\right\} .
$$

The space $\mathscr{A}(\sigma)$ is the space of spline function of degree 3 (or order 4) having knots at $\pi(\sigma)$. Assume $r(t)$ and $p(t)$ are continuous; $r_{\sigma}(t)=r\left(a_{k}\right)$ if $t \in\left[a_{k}, a_{k+1}\right)$ and $r_{\sigma}(b)=r\left(a_{n-1}\right) ; p_{\sigma}(t)$ likewise defined and $J(x ; \sigma)=\int_{a}^{b}\left[r_{\sigma}(t) x^{\prime \prime 2}-p_{\sigma}(t) x^{2}\right] d t$ defined on the space $\mathscr{A}(\sigma)$. Let $\mathscr{H}(\lambda)$ denote the subspace of $\mathscr{A}(\sigma)$ such that $x$ in $\mathscr{H}(\lambda)$ implies $x(t)=0$ on $\lambda \leqq t \leqq b$. Furthermore, let $\mathscr{B}(\mu)=\mathscr{H}(\lambda) \cap \mathscr{A}(\sigma)$.

Since the space $\mathscr{A}(\sigma)$ for $\sigma \neq 0$ has a basis, each element of which has support on at most 4 intervals, the problem of finding $s(\lambda, \sigma)$ and $n(\lambda, \sigma)$ reduces to that of finding the number of negative and zero eigenvalues of a real, symmetric matrix $D_{i j}(\lambda, \sigma)$ which has nonzero entries only if $|i-j| \leqq 3$. For fixed $\sigma$ the function $s(\lambda, \sigma)$ can be found by a modification of the usual Sturm sequence arguments; it represents the number of negative eigenvalues of the matrix $D_{i j}(\lambda, \sigma)$. Note that for fixed $\sigma, D(\lambda, \sigma)$ is "expanding" in $\lambda$, and is of order $k \times k$ if $a_{k} \leqq \lambda<a_{k+1}$.

The approximation results in Theorems 1, 2, 3 and Theorems 5, 6, 7 hold since the extremal properties of splines can be used to show that conditions (1) and (2) are satisfied (see [5]).

\section{REFERENCES}

1. J. Gregory, An approximation theory for elliptic quadratic forms on Hilbert spaces: Application to the eigenvalue problem for compact quadratic forms, Pacific J. Math. 37 (1970), 383-395.

2. — An approximation theory for focal points and focal intervals, Proc. Amer. Math. Soc. 32 (1972), 477-483. 
3. M. R. Hestenes, Applications of the theory of quadratic forms in Hilbert space in the calculus of variations, Pacific J. Math. 1 (1951), 525-581. MR 13, 759.

4. E. Y. Mikami, Focal points in a control problem, Pacific J. Math. 35 (1970), 473-485. MR 43 \#6800.

5. I. J. Schoenberg, Spline interpolation and the higher derivatives, Proc. Nat. Acad. Sci. U.S.A. 51 (1964), 24-28. MR 28 \#3278.

Department of Mathematics, University of Alberta, Edmonton, Alberta, CANADA

Current address: Department of Mathematics, Southern Illinois University, Carbondale, Illinois 62901 